IJMMS 29:1 (2002) 31-42

PII. S0161171202004982

http://ijmms.hindawi.com

(c) Hindawi Publishing Corp.

\title{
INTERACTION BETWEEN LINE CRACKS IN AN ORTHOTROPIC LAYER
}

\author{
SUBIR DAS
}

Received 19 April 2000

\begin{abstract}
We deal with the interaction between three coplanar Griffith cracks located symmetrically in the mid plane of an orthotropic layer of finite thickness $2 h$. The Fourier transform technique is used to reduce the elastostatic problem to the solution of a set of integral equations which have been solved by using the finite Hilbert transform technique and Cooke's result. The analytical expressions for the stress intensity factors at the crack tips are obtained for large $h$. Numerical values of the interaction effect have been computed for and results show that interaction effects are either shielding or amplification depending on the location of each crack with respect to each other and crack tip spacing as well as the thickness of the layer.
\end{abstract}

2000 Mathematics Subject Classification: 74A10, 74A50, 45E05.

1. Introduction. In material structures, pre-existing cracks interact to form major cracks leading fracture. Thus the study of interacting cracks subjected to a given set of external loads become extremely important for the purpose of design and safe-life prediction of material structures.

Problems with Griffith cracks in orthotropic elastic materials were considered by Erdogan et al. [7], Dhaliwal [6], Satpathy and Parhi [18], Piva and Viola [13], Cinar and Erdogan [3], Lowengrub and Srivastava [11], De and Patra [5], Kassir and Tse [9], and others. Analytical studies of crack interaction problems can be found in Sneddon and Lowengrub [19], Rose [14], Lam et al. [10], Brencich and Carpinteri [1], Chudnovsky and Kachanov [2], Rubinstein [16], Horii and Nasser [8], and so forth. The interaction between the elliptic cracks has been studied by Roy and Saha [15] and Murakami and Nishitani [12], and others.

In the present paper, the interaction between three coplanar Griffith cracks in an orthotropic layer of finite depth $2 h$ has been investigated. The resulting mixed boundary value problem is reduced to the solution of a set of integral equations which have been further reduced to the solution of an integro-differential equation. An iterative procedure is adopted to get the approximate analytical expressions for the stress intensity factors by retaining terms up to order of $h^{-2}$ for large $h$. Numerical results for the stress intensity factors at the crack tips and the interaction of the outer cracks on the central one and conversely through stress magnification factors have been calculated. Graphical plots of these results are also presented for illustrations.

2. Formulation of the problem. Consider the plane elastostatic problem of the coplanar Griffith cracks of finite lengths situated in the mid-plane of an infinite orthotropic strip of thickness $2 h$. Under the assumption of plane strain in an orthotropic 
medium, the displacement equations of motion are

$$
\begin{aligned}
& C_{11} \frac{\partial^{2} u}{\partial x^{2}}+C_{66} \frac{\partial^{2} u}{\partial y^{2}}+\left(C_{12}+C_{66}\right) \frac{\partial^{2} v}{\partial x \partial y}=0 \\
& C_{22} \frac{\partial^{2} v}{\partial y^{2}}+C_{66} \frac{\partial^{2} v}{\partial x^{2}}+\left(C_{12}+C_{66}\right) \frac{\partial^{2} u}{\partial x \partial y}=0 .
\end{aligned}
$$

The stress displacement relations are

$$
\begin{aligned}
& \sigma_{x x}=C_{11} \frac{\partial u}{\partial x}+C_{12} \frac{\partial v}{\partial y}, \\
& \sigma_{y y}=C_{22}\left(\frac{\partial v}{\partial y}+\frac{\partial u}{\partial x}\right), \\
& \sigma_{x y}=C_{66}\left(\frac{\partial u}{\partial y}+\frac{\partial v}{\partial x}\right) .
\end{aligned}
$$

The problem is symmetric with respect to $y=0$. Assuming the cracks $|x|<b, c<$ $|x|<1, y=0$ opened by normal pressure $p(x)$, the boundary conditions for the problem to be studied are

$$
\begin{aligned}
\sigma_{y y}(x, 0) & =-p(x), \quad|x|<b, c<|x|<1, \\
v(x, 0) & =0, \quad b \leq|x| \leq c,|x| \geq 1, \\
\sigma_{x y}(x, 0) & =0, \quad|x|<\infty
\end{aligned}
$$

and on $y=h$

$$
\begin{array}{ll}
u(x, h)=0, & |x|<\infty, \\
v(x, h)=0, & |x|<\infty .
\end{array}
$$

In addition all the components of stress and displacement vanish at the remote distances from the cracks.

3. Solution of the problem. The appropriate solution of (2.1) can be taken as

$$
\begin{aligned}
& u(x, y)=\int_{0}^{\infty} A(s, y) \sin s x d s, \\
& v(x, y)=\int_{0}^{\infty} B(s, y) \cos s x d s,
\end{aligned}
$$

where $A$ and $B$ are functions of $y$ satisfying the equations

$$
\begin{aligned}
& C_{11} s^{2} A-C_{66} \frac{d^{2} A}{d y^{2}}+\left(C_{12}+C_{66}\right) s \frac{d B}{d y}=0, \\
& C_{66} s^{2} B-C_{22} \frac{d^{2} B}{d y^{2}}-\left(C_{12}+C_{66}\right) s \frac{d A}{d y}=0,
\end{aligned}
$$

which has the solution for the layer as

$$
\begin{aligned}
& A(s, y)=A_{1}(s) \operatorname{ch}\left(\gamma_{1} s y\right)+A_{2}(s) \operatorname{ch}\left(\gamma_{2} s y\right)+C_{1}(s) \operatorname{sh}\left(\gamma_{1} s y\right)+C_{2}(s) \operatorname{sh}\left(\gamma_{2} s y\right), \\
& B(s, y)=B_{1}(s) \operatorname{sh}\left(\gamma_{1} s y\right)+B_{2}(s) \operatorname{sh}\left(y_{2} s y\right)+D_{1}(s) \operatorname{ch}\left(\gamma_{1} s y\right)+D_{2}(s) \operatorname{ch}\left(\gamma_{2} s y\right),
\end{aligned}
$$


in which $\gamma_{1}$ and $\gamma_{2}\left(<\gamma_{1}\right)$ are positive roots of the equation

$$
C_{66} C_{22} \gamma^{4}+\left[\left(C_{12}+C_{66}\right)^{2}-C_{11} C_{22}-C_{66}^{2}\right] \gamma^{2}+C_{11} C_{66}=0,
$$

and $B_{j}(s), D_{j}(s)$ are related to $A_{j}(s)$ and $C_{j}(s)$ by

$$
B_{j}(s)=-\frac{\alpha_{j}}{\gamma_{j}} A_{j}(s), \quad D_{j}(s)=-\frac{\alpha_{j}}{\gamma_{j}} C_{j}(s),
$$

where

$$
\alpha_{j}=\frac{\left(C_{11}-\gamma_{j}^{2} C_{66}\right)}{\left(C_{12}+C_{66}\right)}, \quad j=1,2
$$

The expressions for the stresses are

$$
\begin{aligned}
\sigma_{x y}(x, y)=C_{66} \int_{0}^{\infty}\left[\frac{\beta_{1}}{\gamma_{1}} A_{1}(s) \operatorname{sh}\left(\gamma_{1} s y\right)+\frac{\beta_{2}}{\gamma_{2}} A_{2}(s) \operatorname{sh}\left(\gamma_{2} s y\right)\right. \\
\left.+\frac{\beta_{1}}{\gamma_{1}} C_{1}(s) \operatorname{ch}\left(\gamma_{1} s y\right)+\frac{\beta_{2}}{\gamma_{2}} C_{2}(s) \operatorname{ch}\left(\gamma_{2} s y\right)\right] s \sin s x d s, \\
\sigma_{y y}(x, y)=\int_{0}^{\infty}\left[\left(C_{12}-C_{22} \alpha_{1}\right) A_{1}(s) \operatorname{ch}\left(\gamma_{1} s y\right)+\left(C_{12}-C_{22} \alpha_{2}\right) A_{2}(s) \operatorname{ch}\left(\gamma_{2} s y\right)\right. \\
\left.+\left(C_{12}-C_{22} \alpha_{1}\right) C_{1}(s) \operatorname{sh}\left(\gamma_{1} s y\right)+\left(C_{12}-C_{22} \alpha_{2}\right) C_{2}(s) \operatorname{sh}\left(\gamma_{2} s y\right)\right] \cos s x d s .
\end{aligned}
$$

Applying (2.5), we obtain

$$
C_{2}(s)=-\frac{\beta_{1} \gamma_{2}}{\beta_{2} \gamma_{1}} C_{1}(s)
$$

with

$$
\beta_{j}=\alpha_{j}+\gamma_{j}^{2}, \quad j=1,2
$$

The boundary conditions (2.6) and (2.7) in conjunction with (3.8) give rise to

$$
A_{1}(s)=\delta_{1}(s h) C_{1}(s), \quad A_{2}(s)=\delta_{2}(s h) C_{1}(s),
$$

whose $\delta_{1}(s h), \delta_{2}(s h)$ are known functions and are given by

$$
\begin{aligned}
\delta_{1}(\operatorname{sh})= & \frac{\alpha_{2} \beta_{1} / \beta_{2} \gamma_{1}+\left(\alpha_{2} / \gamma_{2}\right) \operatorname{sh}\left(\gamma_{1} \operatorname{sh}\right) \operatorname{sh}\left(\gamma_{2} \operatorname{sh}\right)-\left(\alpha_{1} / \gamma_{1}\right) \operatorname{ch}\left(\gamma_{1} \operatorname{sh}\right) \operatorname{ch}\left(\gamma_{2} \operatorname{sh}\right)}{\left(\alpha_{1} / \gamma_{1}\right) \operatorname{sh}\left(\gamma_{1} \operatorname{sh}\right) \operatorname{ch}\left(\gamma_{2} \operatorname{sh}\right)-\left(\alpha_{2} / \gamma_{2}\right) \operatorname{sh}\left(\gamma_{2} \operatorname{sh}\right) \operatorname{ch}\left(\gamma_{1} \operatorname{sh}\right)}, \\
\delta_{2}(\operatorname{sh})= & \frac{\alpha_{1} / \gamma_{1}+\left(\alpha_{1} \beta_{1} \gamma_{2} / \beta_{2} \gamma_{1}^{2}\right) \operatorname{sh}\left(\gamma_{1} \operatorname{sh}\right) \operatorname{sh}\left(\gamma_{2} \operatorname{sh}\right)}{\left(\alpha_{1} / \gamma_{1}\right) \operatorname{sh}\left(\gamma_{1} \operatorname{sh}\right) \operatorname{ch}\left(\gamma_{2} \operatorname{sh}\right)-\left(\alpha_{2} / \gamma_{2}\right) \operatorname{sh}\left(\gamma_{2} \operatorname{sh}\right) \operatorname{ch}\left(\gamma_{1} \operatorname{sh}\right)} \\
& -\frac{\left(\alpha_{2} \beta_{1} / \beta_{2} \gamma_{1}\right) \operatorname{ch}\left(\gamma_{1} \operatorname{sh}\right) \operatorname{ch}\left(\gamma_{2} \operatorname{sh}\right)}{\left(\alpha_{1} / \gamma_{1}\right) \operatorname{sh}\left(\gamma_{1} \operatorname{sh}\right) \operatorname{ch}\left(\gamma_{2} \operatorname{sh}\right)-\left(\alpha_{2} / \gamma_{2}\right) \operatorname{sh}\left(\gamma_{2} \operatorname{sh}\right) \operatorname{ch}\left(\gamma_{1} \operatorname{sh}\right)} .
\end{aligned}
$$


The boundary conditions (2.3) and (2.4) finally yield the following integral equations:

$$
\begin{gathered}
\int_{0}^{\infty} s C_{1}(s)[1+M(s h)] \cos s x d s=-\frac{1}{D} p(x), \quad 0<x<b, c<x<1, \\
\int_{0}^{\infty} C_{1}(s) \cos s x d s=0, \quad b<x<c, x>1,
\end{gathered}
$$

where

$$
\begin{gathered}
M(s h)=-\left[1+\frac{\left(C_{12}-C_{22} \alpha_{1}\right) \delta_{1}(s h)+\left(C_{12}-C_{22} \alpha_{2}\right) \delta_{2}(s h)}{D}\right], \\
D=\left(C_{12}-C_{22} \alpha_{1}\right)-\frac{\beta_{1} \gamma_{2}}{\beta_{2} \gamma_{1}}\left(C_{12}-C_{22} \alpha_{2}\right) .
\end{gathered}
$$

It should be noted that $M(s h) \rightarrow 0$ as $h \rightarrow \infty$.

Setting

$$
C_{1}(s)=\frac{1}{s} \int_{0}^{b} h(t) \sin s t d t+\frac{1}{s} \int_{c}^{1} g\left(u^{2}\right) \sin s u d u,
$$

where $h(t)$ and $g\left(u^{2}\right)$ are the unknown functions. Using the result

$$
\int_{0}^{\infty} \frac{\sin s t \cos s x}{s} d s= \begin{cases}\frac{\pi}{2}, & t>x \\ 0, & t<x\end{cases}
$$

it is found that (3.13) is identically satisfied if

$$
\int_{c}^{1} g\left(u^{2}\right) d u=0
$$

Further, using the result

$$
\int_{0}^{\infty} \frac{\sin s u \sin s x}{s} d s=\frac{1}{2} \log \left|\frac{u+x}{u-x}\right|
$$

equation (3.12) under (3.15) leads to

$$
\begin{aligned}
\frac{d}{d x} \int_{0}^{b} h(t) \log \left|\frac{t+x}{t-x}\right| d t & +\frac{d}{d x} \int_{c}^{1} g\left(u^{2}\right) \log \left|\frac{u+x}{u-x}\right| d u \\
& +\frac{d}{d x} \int_{0}^{b} h(t) d t \int_{0}^{\infty} s^{-1} M(s h) \sin s t \sin s x d s \\
& +\frac{d}{d x} \int_{c}^{1} g\left(u^{2}\right) d u \int_{0}^{\infty} s^{-1} M(s h) \sin s u \sin s x d s \\
& =-\frac{2}{D} p(x), \quad 0<x<b, c<x<1 .
\end{aligned}
$$


Now assuming

$$
\begin{gathered}
h(t)=h_{0}(t)+\frac{1}{h^{2}} h_{1}(t)+O\left(\frac{1}{h^{4}}\right), \\
g\left(u^{2}\right)=g_{0}\left(u^{2}\right)+\frac{1}{h^{2}} g_{1}\left(u^{2}\right)+O\left(\frac{1}{h^{4}}\right),
\end{gathered}
$$

for large $h$, integral equation (3.19) reduces to

$$
\begin{aligned}
& \frac{d}{d x} \int_{0}^{b} h_{0}(t) \log \left|\frac{t+x}{t-x}\right| d t+2 \int_{c}^{1} \frac{u g_{0}\left(u^{2}\right) d u}{u^{2}-x^{2}}=-\frac{2}{D} p(x) \\
& \frac{d}{d x} \int_{0}^{b} h_{1}(t) \log \left|\frac{t+x}{t-x}\right| d t+2 \int_{c}^{1} \frac{u g_{1}\left(u^{2}\right) d u}{u^{2}-x^{2}} \\
& \quad=-2 P\left[\int_{0}^{b} t h_{0}(t) d t+\int_{c}^{1} u g_{0}\left(u^{2}\right) d u\right], \quad 0<x<b, c<x<1
\end{aligned}
$$

with

$$
\int_{c}^{1} g_{i}\left(u^{2}\right) d u=0, \quad i=0,1
$$

where

$$
\begin{aligned}
& P= \frac{1}{D\left(\left(\alpha_{1}\right) /\left(\gamma_{1}\right)-\left(\alpha_{2}\right) /\left(\gamma_{2}\right)\right)} \\
& \times\left[\frac{1}{2 \gamma_{1}^{2}}\left(C_{12}-C_{22} \alpha_{1}\right)\left(\frac{\alpha_{1}}{\gamma_{1}}+\frac{\alpha_{2}}{\gamma_{2}}\right)+\frac{\beta_{1}}{2 \beta_{2} \gamma_{1} \gamma_{2}}\left(C_{12}-C_{22} \alpha_{2}\right)\left(\frac{\alpha_{1}}{\gamma_{1}}+\frac{\alpha_{2}}{\gamma_{2}}\right)\right. \\
&\left.\quad+\frac{\alpha_{2} \beta_{1}}{\beta_{2} \gamma_{1}}\left\{\left(C_{12}-C_{22} \alpha_{1}\right)-\frac{\alpha_{1} \beta_{2}}{\alpha_{2} \beta_{1}}\left(C_{12}-C_{22} \alpha_{2}\right)\right\} \times \frac{1}{\left(\gamma_{1}+\gamma_{2}\right)^{2}}\right] .
\end{aligned}
$$

Rewriting equation (3.21) as

$$
\int_{0}^{b} h_{0}(t) \log \left|\frac{t+x}{t-x}\right| d t=\pi F_{1}(x)
$$

where

$$
F_{1}(x)=-\frac{1}{\pi} \int_{0}^{x}\left[\frac{2}{D} p(y)+\int_{c}^{1} \frac{2 u g_{0}\left(y^{2}\right)}{u^{2}-y^{2}} d u\right] d y
$$

and using Cooke's result [4], the solution of the integral equation is found to be

$$
h_{0}(t)=\frac{4}{\pi^{2} D} \frac{t}{\sqrt{b^{2}-t^{2}}} P_{1}(t)-\frac{2}{\pi} \frac{t}{\sqrt{b^{2}-t^{2}}} \int_{c}^{1} \frac{\sqrt{u^{2}-b^{2}} g_{0}\left(y^{2}\right)}{u^{2}-t^{2}} d u
$$

where

$$
P_{1}(t)=\int_{0}^{b} \frac{\sqrt{b^{2}-x^{2}}}{x^{2}-t^{2}} p(x) d x
$$


then the integral equation for $g_{0}\left(u^{2}\right)$ is derived as

$$
\int_{c}^{1} \frac{\sqrt{u^{2}-b^{2}} g_{0}\left(u^{2}\right) d u}{u^{2}-x^{2}}=-F_{2}(x)
$$

with

$$
F_{2}(x)=\frac{\sqrt{x^{2}-b^{2}}}{x}\left[\frac{1}{D} p(x)+\frac{4}{\pi^{2} D} \int_{0}^{b} \frac{t^{2} P_{1}(t)}{\sqrt{b^{2}-t^{2}}\left(t^{2}-x^{2}\right)} d t\right] .
$$

Now using Hilbert transform technique (see Sarkar et al. [17]), the solution to (3.27) is found to be

$$
\begin{aligned}
g_{0}\left(u^{2}\right)= & \frac{2 u}{\pi \sqrt{\left(u^{2}-c^{2}\right)\left(1-u^{2}\right)\left(u^{2}-b^{2}\right)}} \int_{c}^{1} \frac{\sqrt{x^{2}\left(x^{2}-c^{2}\right)\left(1-x^{2}\right)}}{x^{2}-u^{2}} \\
& +\frac{u k_{1}}{\sqrt{\left(u^{2}-c^{2}\right)\left(1-u^{2}\right)\left(u^{2}-b^{2}\right)}}
\end{aligned}
$$

where $k_{1}$ is an unknown constant to be determined from (3.23). Then the closed form of the expression for $h_{0}(t)$ may be obtained from (3.27) when the use of (3.29) is made there. Again applying the same procedure and using the above result, analytical expressions of $h_{1}(t)$ and $g_{1}\left(u^{2}\right)$ may be derived. As a particular case of the problem setting $p(x)=p$, a constant, analytical expressions for $h_{j}(t)$ and $g_{j}\left(u^{2}\right), j=0,1$ are obtained as

$$
\begin{aligned}
h_{0}(t) & =-\frac{2 p}{D} \sqrt{\frac{t^{2}\left(c^{2}-t^{2}\right)}{\left(b^{2}-t^{2}\right)\left(1-t^{2}\right)}}+\frac{t k_{1}}{\sqrt{\left(b^{2}-t^{2}\right)\left(c^{2}-t^{2}\right)\left(1-t^{2}\right)}}, \\
h_{1}(t) & =-\frac{2 P R}{\pi} \sqrt{\frac{t^{2}\left(c^{2}-t^{2}\right)}{\left(b^{2}-t^{2}\right)\left(1-t^{2}\right)}}-\frac{t k_{2}}{\sqrt{\left(b^{2}-t^{2}\right)\left(c^{2}-t^{2}\right)\left(1-t^{2}\right)}}, \\
g_{0}\left(u^{2}\right) & =-\frac{2 p}{D} \sqrt{\frac{u^{2}\left(u^{2}-c^{2}\right)}{\left(u^{2}-b^{2}\right)\left(1-u^{2}\right)}}+\frac{u k_{1}}{\sqrt{\left(u^{2}-b^{2}\right)\left(u^{2}-c^{2}\right)\left(1-u^{2}\right)}}, \\
g_{1}\left(u^{2}\right) & =-\frac{2 P R}{\pi} \sqrt{\frac{u^{2}\left(u^{2}-c^{2}\right)}{\left(u^{2}-b^{2}\right)\left(1-u^{2}\right)}}+\frac{u k_{2}}{\sqrt{\left(u^{2}-b^{2}\right)\left(u^{2}-c^{2}\right)\left(1-u^{2}\right)}},
\end{aligned}
$$

where

$$
\begin{aligned}
R & =-\frac{2 p}{D}\left[I_{0}^{b}+I_{c}^{1}\right]-K_{1}\left[J_{0}^{b}-J_{c}^{1}\right], \\
I_{m}^{n} & =\int_{m}^{n} \frac{t^{2} \sqrt{c^{2}-t^{2}}}{\sqrt{\left(b^{2}-t^{2}\right)\left(1-t^{2}\right)}} d t, \\
J_{m}^{n} & =\int_{m}^{n} \frac{t^{2} d t}{\sqrt{\left(b^{2}-t^{2}\right)\left(c^{2}-t^{2}\right)\left(1-t^{2}\right)}}, \\
k_{j} & =A_{j}\left[\left(1-b^{2}\right) \frac{E}{F}-\left(c^{2}-b^{2}\right)\right], \quad j=1,2,
\end{aligned}
$$


with

$$
A_{1}=\frac{2 p}{D}, \quad A_{2}=\frac{2 P R}{\pi} .
$$

In the above, $F=F(\pi / 2, q)$ and $E=E(\pi / 2, q)$ are the elliptic integrals of first and second kinds, respectively, and $q=\sqrt{\left(1-c^{2}\right) /\left(1-b^{2}\right)}$. The stress intensity factors $K_{b}$, $K_{c}$, and $K_{1}$ at the crack tips $x=b, x=c$, and $x=1$ of the cracks are found to be

$$
\begin{aligned}
K_{b} & =\lim _{x \rightarrow c+} \sqrt{2(x-b)} \sigma_{y y}(x, 0)=p \sqrt{\frac{b\left(1-b^{2}\right)}{c^{2}-b^{2}}} \frac{E}{F}\left[1-\frac{2 P}{\pi} \frac{W}{h^{2}}\right]+O\left(h^{-4}\right), \\
K_{c} & =\lim _{x \rightarrow c-} \sqrt{2(c-x)} \sigma_{y y}(x, 0) \\
& =p \sqrt{\frac{c}{\left(c^{2}-b^{2}\right)\left(1-c^{2}\right)}}\left[\left(1-b^{2}\right) \frac{E}{F}-\left(c^{2}-b^{2}\right)\right]\left[1-\frac{2 P}{\pi} \frac{W}{h^{2}}\right]+O\left(h^{-4}\right), \\
K_{1} & =\lim _{x \rightarrow 1-} \sqrt{2(x-1)} \sigma_{y y}(x, 0)=p \sqrt{\frac{1-b^{2}}{1-c^{2}}}\left[1-\frac{E}{F}\right]\left[1-\frac{2 P}{\pi} \frac{W}{h^{2}}\right]+O\left(h^{-4}\right),
\end{aligned}
$$

where

$$
W=\left[I_{0}^{b}+I_{c}^{1}+\left\{\left(1-b^{2}\right) \frac{E}{F}-\left(c^{2}-b^{2}\right)\right\}\left(J_{0}^{b}-J_{c}^{1}\right)\right] .
$$

The stress magnification factors $M_{b}, M_{c}$, and $M_{1}$ at the crack tips $x=b, x=c$, and $x=1$ are defined as $M_{b}=K_{b} / K_{b}^{*}, M_{c}=K_{c} / K_{c}^{*}, M_{1}=K_{1} / K_{1}^{*}$, where $K_{b}^{*}$ is the stress intensity factor at $x=b$ due to the presence of the central crack only and $K_{c}^{*}, K_{1}^{*}$ are the stress intensity factors at $x=c, x=1$, respectively, due to the presence of outer cracks only and these are given by

$$
K_{b}^{*}=p \sqrt{b} \frac{E}{F}\left[1-\frac{2 P}{\pi} \frac{Q_{1}}{h^{2}}\right]+O\left(h^{-4}\right)
$$

where

$$
\begin{aligned}
& Q_{1}=I_{0}^{b}+\left(1-b^{2}\right)\left[\frac{E}{F}-1\right] J_{0}^{b}, \\
& K_{c}^{*}=\frac{p}{\sqrt{c\left(1-c^{2}\right)}}\left[\frac{E}{F}-c^{2}\right]\left[1-\frac{2 P}{\pi} \frac{Q_{2}}{h^{2}}\right]+O\left(h^{-4}\right), \\
& K_{1}^{*}=\frac{p}{\sqrt{1-c^{2}}}\left[1-\frac{E}{F}\right]\left[1-\frac{2 P}{\pi} \frac{Q_{2}}{h^{2}}\right]+O\left(h^{-4}\right), \\
& Q_{2}=I_{c}^{1}-\left[\frac{E}{F}-c^{2}\right] J_{c}^{1} .
\end{aligned}
$$

4. Numerical results and discussion. As a particular case of the problem, the orthotropic material is considered to be $\alpha$-Uranium. Values of the material constants are taken as

\begin{tabular}{ccccc}
\hline & $C_{11}$ & $C_{22}$ & $C_{12}$ & $C_{66}$ \\
\hline$\alpha$-Uranium & 21.47 & 19.86 & 4.65 & 7.43 \\
\hline
\end{tabular}




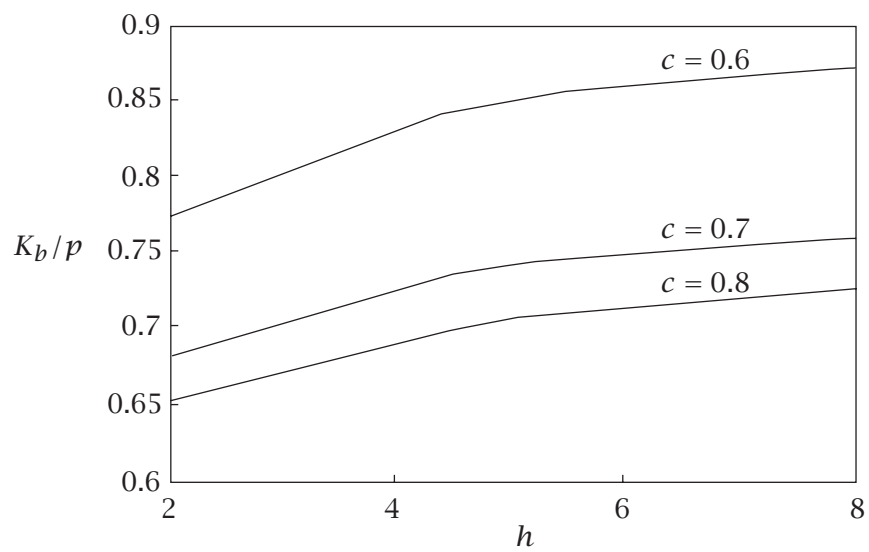

FIGURE 4.1. Plot of $K_{b} / p$ versus $h$ at $b=0.5$.

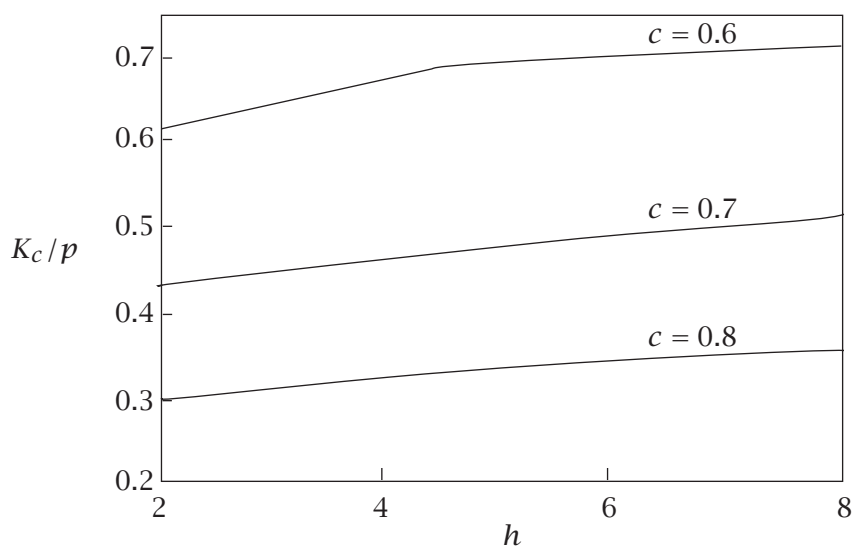

FIGURE 4.2. Plot of $K_{c} / p$ versus $h$ at $b=0.5$.

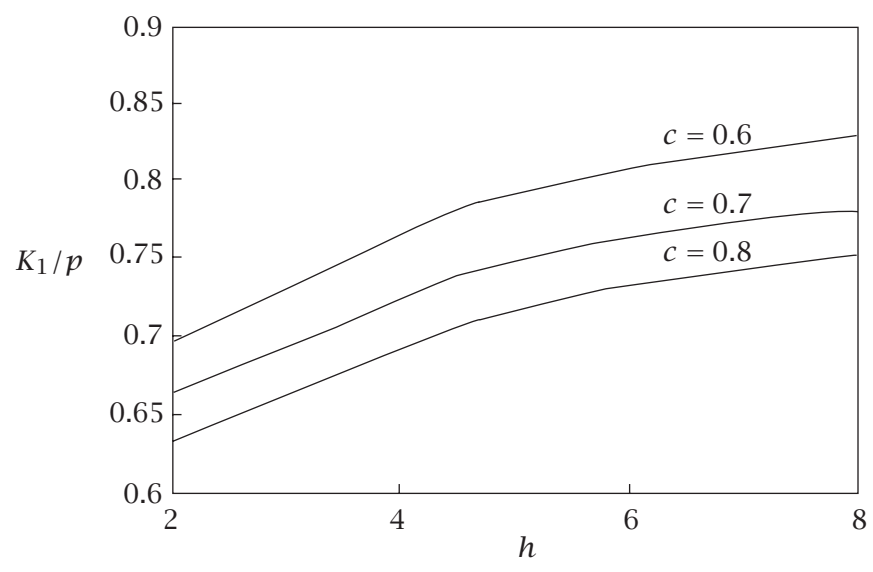

FIGURE 4.3. Plot of $K_{1} / p$ versus $h$ at $b=0.5$. 


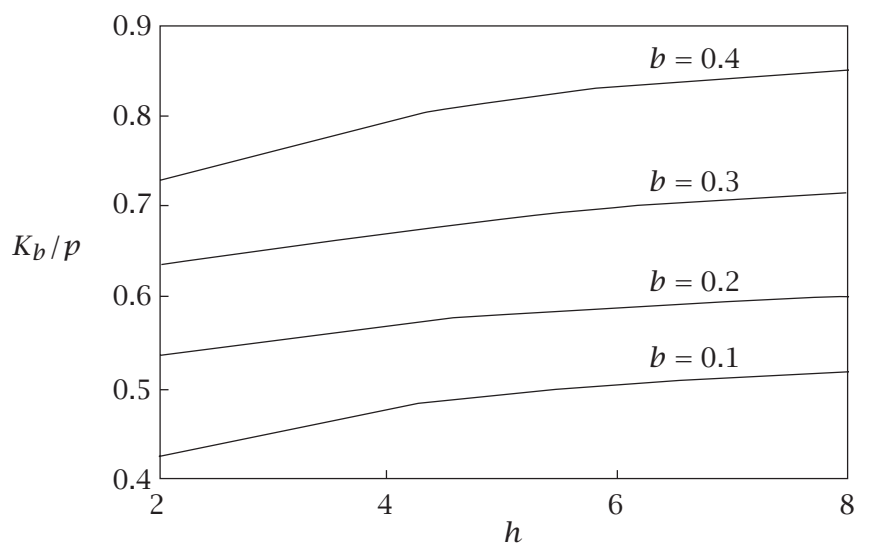

FIGURE 4.4. Plot of $K_{b} / p$ versus $h$ at $c=0.5$.

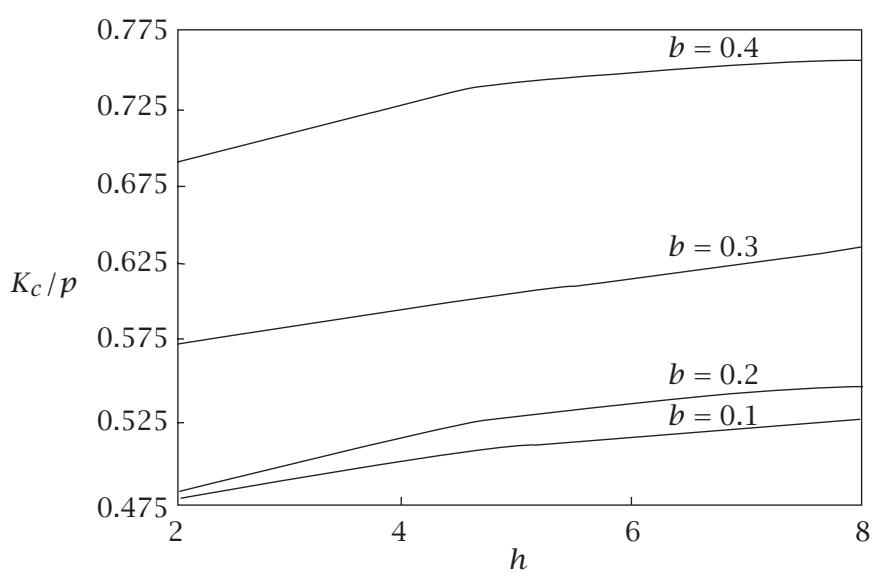

FIGURE 4.5. Plot of $K_{c} / p$ versus $h$ at $c=0.5$.

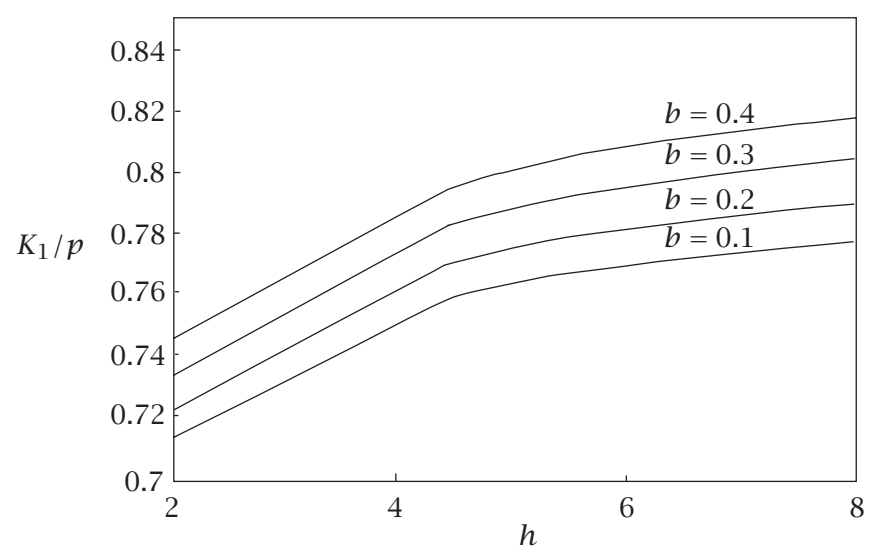

FIGURE 4.6. Plot of $K_{1} / p$ versus $h$ at $c=0.5$. 


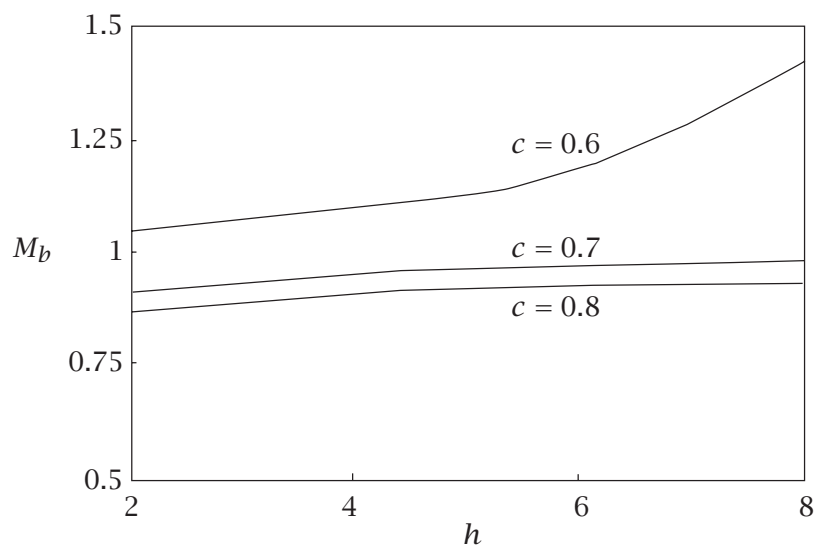

FIgURE 4.7. Plot of $M_{b}$ versus $h$ at $b=0.5$.

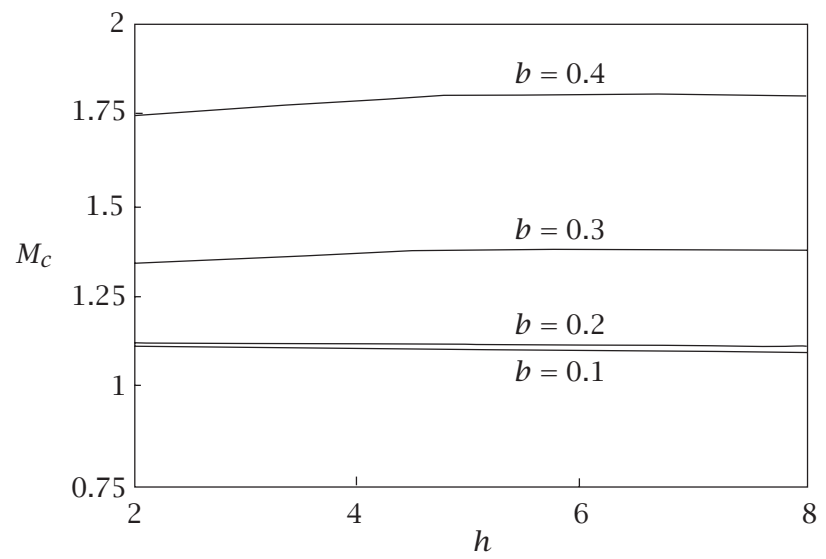

Figure 4.8. Plot of $M_{\mathcal{C}}$ versus $h$ at $c=0.5$.

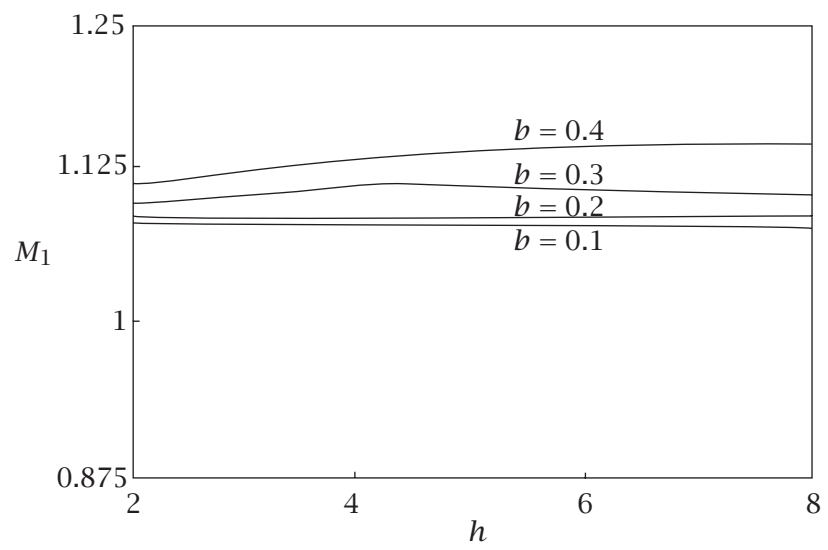

FIGURE 4.9. Plot of $M_{1}$ versus $h$ at $c=0.5$. 
The stress intensity factors $K_{b}, K_{c}$, and $K_{1}$ at the tips of the cracks opened by a constant pressure $p$ have been plotted against $h$. Keeping the central crack length fixed $(b=0.5)$, stress intensity factors at the tips of the central and outer cracks have been plotted against the depth $h$ for different outer crack lengths $c=0.6,0.7,0.8$. It is observed from Figures 4.1, 4.2, and 4.3, that the stress intensity factors $K_{b}, K_{c}$, and $K_{1}$ decrease with the decrease in outer crack length, that is, with the increase in the distances between the inner and outer cracks and also with the decrease in $h$ for different outer crack lengths.

Again, on keeping the outer crack length fixed at $c=0.5$, it is observed from Figures $4.4,4.5$, and 4.6 that the stress intensity factors increase with an increase in both $h$ and $b$.

Regarding interactions between the central and the external cracks, plots of stress magnification factors through Figures $4.7,4.8$, and 4.9 have been made. It is observed from Figure 4.7 that on keeping the central crack length fixed at $b=0.5$ the stress magnification factor $M_{b}$ decreases with a decrease in the outer crack lengths and increase with an increase in $h$.

In this case the interaction effect of outer crack on central crack is a mixture of amplification and shielding. When the outer crack is relatively smaller $(c=0.8)$ and depth of the layer is narrower $(h=2)$ the shielding effect is maximum. Maximum amplification is attained at the crack tip nearest to the adjacent central crack $(c=0.6)$ and at large depth $h=8$.

It is seen from Figures 4.8 and 4.9 that the stress magnifications $M_{c}$ and $M_{1}$ increase with an increase in the central crack length and also with an increase in $h$ when the outer crack length is fixed at $c=0.5$. It is clearly seen that the effect of central crack on the outer cracks is amplification. At both the ends amplifications are maximum when the central crack length and depth of the layer become large $(b=0.4, h=8)$.

5. Conclusion. We have seen that the central and outer cracks interaction effect is a mixture of shielding and amplification or simply amplification depending on the length of the cracks and the depth of the layer. When the outer crack is smaller, there is possible crack arrest of the central crack. When the outer crack is broad, there is a propagation tendency of the central crack towards the outer crack. Again as the central crack length increases, the propagation tendency of outer crack at both ends increase due to increase of amplifications.

\section{REFERENCES}

[1] A. Brencich and A. Carpinteri, Interaction of a main crack with ordered distributions of microcracks: a numerical technique by displacement discontinuity boundary elements, Internat. J. Fracture 76 (1996), 373-389.

[2] A. Chudnovsky and M. Kachanov, Interaction of a crack with a field of microcracks, Internat. J. Engrg. Sci. 21 (1983), 1009-1018.

[3] A. Cinar and F. Erdogan, The crack and wedging problem for an orthotropic strip, Internat. J. Fracture 23 (1983), 83-102.

[4] J. C. Cooke, The solution of some integral equations and their connection with dual integral equations and series, Glasgow Math. J. 11 (1970), 9-20.

[5] J. De and B. patra, Moving Griffith crack in an orthotropic strip, Internat. J. Engrg. Sci. 28 (1990), 809-819. 
[6] R. S. Dhaliwal, Two coplanar cracks in an infinitely long orthotropic elastic strip, Utilitas Math. 4 (1973), 115-128.

[7] F. Erdogan, G. Gupta, and T. S. Cook, Numerical solution of singular integral equations, Mechanics of Fracture, vol. 1, Noordhoff, Leiden, 1973, pp. 368-425.

[8] M. Horii and S. Neman-Nasser, Interacting micro-cracks near the tip in the process zone of a macro-crack, J. Mech. Phys. Solids 35 (1987), 601-629.

[9] M. K. Kassir and S. Tse, Moving Griffith crack in an orthotropic material, Internat. J. Engrg. Sci. 21 (1983), 315-325.

[10] K. Y. Lam, C. Wen, and Z. Phua, Interaction between medium, Engrg. Fracture Mech. 44 (1993), 753-761.

[11] M. Lowengrub and K. N. Srivastava, Two coplanar Griffith cracks in an infinitely long elastic strip, Internat. J. Engrg. Sci. 6 (1968), 425-434.

[12] Y. Murakami and H. Nishitani, Stress intensity factors for interacting two equal semi-elliptic surface cracks in tension, Transaction of JSME 47 (1981), 295-303.

[13] A. Piva and E. Viola, Crack propagating in an orthotropic medium, Engrg. Fracture Mech. 29 (1988), 535-548.

[14] L. R. Rose, Microcrack interaction with a main crack, Internat. J. Fracture 31 (1986), 233242.

[15] A. Roy and T. K. Saha, Interaction of a penny-shaped crack with an elliptic crack, Internat. J. Fracture 73 (1995), 51-65.

[16] A. Rubinstein, Microcrack-microdefect interaction, J. Appl. Math. Mech. 53 (1986), 505510.

[17] J. Sarkar, S. C. Mandal, and M. L. Ghosh, Diffraction of elastic waves by three coplanar Griffith cracks in an orthotropic medium, Internat. J. Engrg. Sci. 33 (1995), 163177.

[18] P. K. Satpathy and H. Parhi, Stresses in an orthotropic strip containing a Griffith crack, Internat. J. Engrg. Sci. 16 (1978), 147-154.

[19] I. N. Sneddon and M. Lowengrub, Crack Problems in the Classical Theory of Elasticity, John Wiley \& Sons, New York, 1969.

Subir DAs: DePartment of MATHEMATics, B.P. PodDAR INSTITUTE of MANAGEMENT AND TECHNOLOGY PODDAR VIHAR, 137, VIP ROAD, CALCUTTA-52, INDIA 


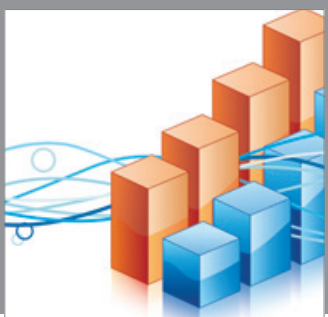

Advances in

Operations Research

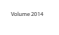

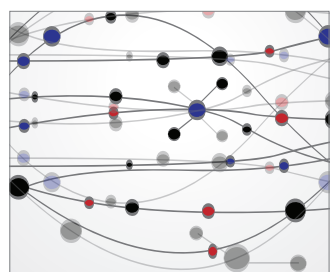

\section{The Scientific} World Journal
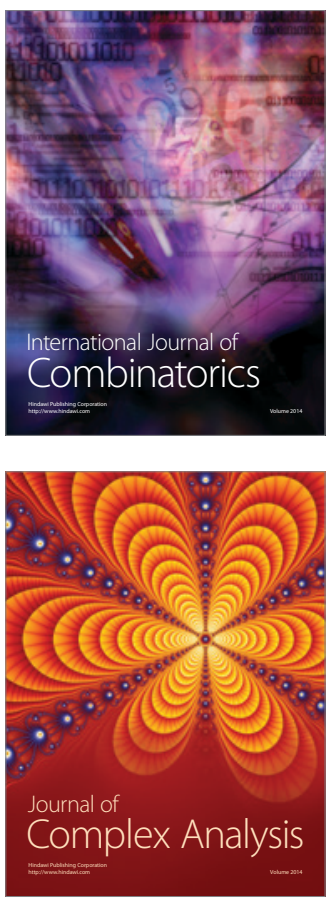

International Journal of

Mathematics and

Mathematical

Sciences
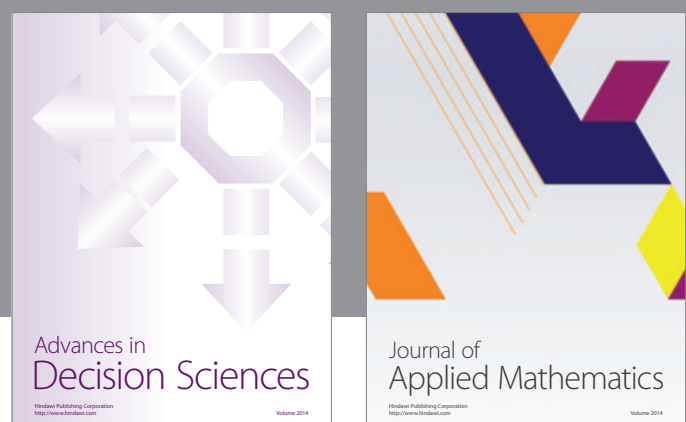

Journal of

Applied Mathematics
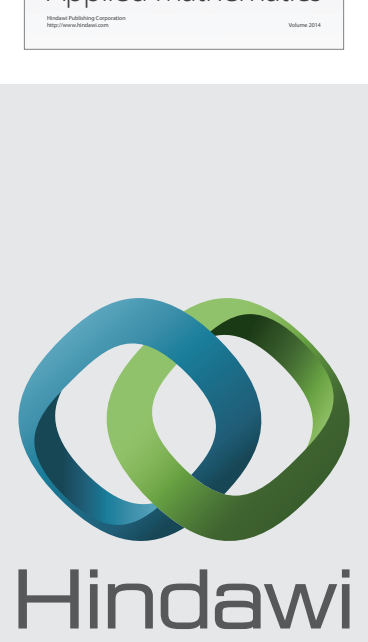

Submit your manuscripts at http://www.hindawi.com
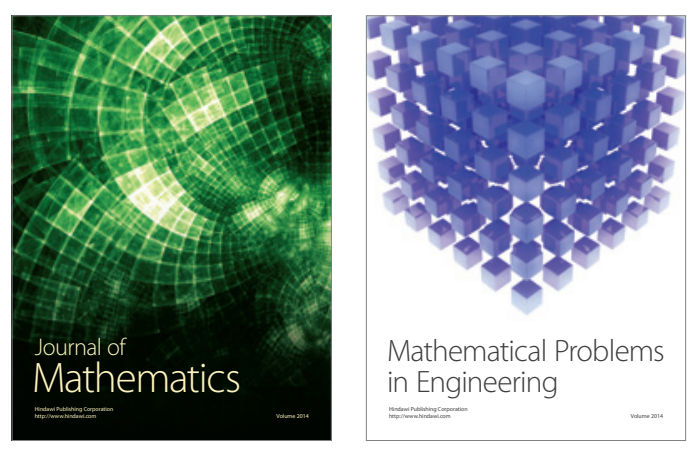

Mathematical Problems in Engineering
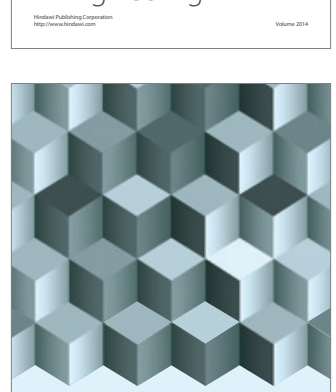

Journal of

Function Spaces
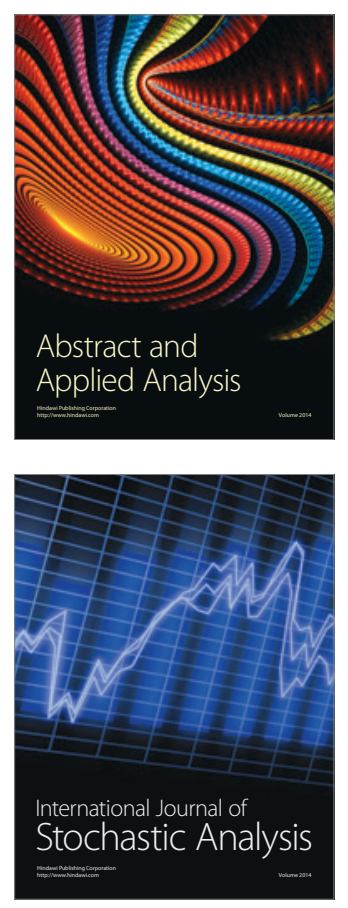

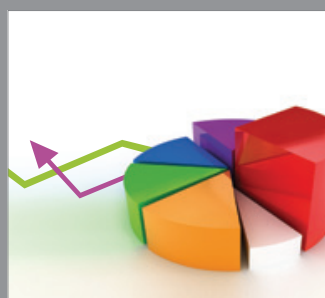

ournal of

Probability and Statistics

Promensencen
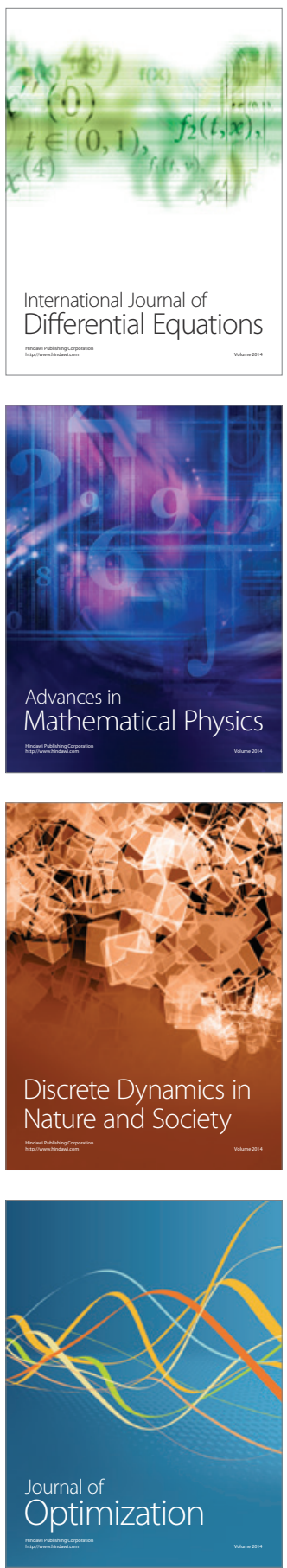